\title{
Fundamental Physical Constants: Input Data and Recommended Values by CODATA 2002
}

\author{
S. G. Karshenboim \\ Mendeleev All-Russian Research Institute of Metrology, St. Petersburg, 190005 Russia \\ Max-Planck-Institut für Quantenoptik, 85748 Garching, Germany
}

\begin{abstract}
The CODATA recommended values of the fundamental physical constants are the most accurate and most reliable constant values applied in different branches of physics. Various questions related to recommendations are considered.

PACS: 02.60.-x; 06.20.Jr

DOI: $10.1134 / \mathrm{S} 1547477108030370$
\end{abstract}

\section{INTRODUCTION}

Computations, whether they are theoretical predictions from first principles or auxiliary calculations required to process an experiment, are certainly of great importance in physics. Physics is impossible without computations. These are based on two essentials: formulas that represent physical laws and various dimensional and dimensionless parameters the numerical values of which define numerical values of the results and which in their turn are determined experimentally. Certain such parameters, like the fine structure constant $\alpha$, the Planck constant $h$, and the electron charge $e$, are of rather universal character and are said to be fundamental constants.

The recommended values of the fundamental physical constants are regularly published by the CODATA (Committee for Data of the ICSU) international working group [1, 2] (see also [3, 4]). As a member of this working group and also being engaged in precision research, I have repeatedly come up against various errors in the use of the values of the fundamental constants. The chief cause of these errors is a certain carelessness and failure to understand what the results of various compilations and processing, such as the recommended values of constants, properly are.

Any reference table, a table of fundamental constants or of other numerous reference data, for example, charge nuclear radii, suggests certain initial data and certain theoretical assumptions and models. The use of data by themselves, taking no account of how these data have been obtained, leads to an inconsistency and eventually produces errors. Having forgotten that some characteristics found experimentally provide a basis for the calculation of a particular constant, one computes this characteristic. Ignoring the fact that the result has been obtained under some assumption, one uses it in a situation when quite another thing is initially assumed. In the computation one employs a few constants, disre- garding the fact that these were computed consistently, and hence their errors are correlated.

To avoid such confusion, it is important to understand the origins of different values and the correlations arising during their calculation. The fundamental constants, as a rule, are computed without resort to model assumptions in the ordinary sense. One is however always confronted with the question of uncertainties in theoretical formulas and of the applicability of some not fully reliable results (for instance, partial calculations, estimates of high-order corrections for which there are conflicting results, etc.). Subsequent analysis may show that some high-order corrections should be corrected and some errors revised.

Working out the recommended values is a rather intricate process, yet all its key steps have been described in the working group's publications $[1,2]$, with critical data analysis being at the heart of them. These publications are an extremely uncommon, if not the only, example of published reference data that comprehensively cover all the used experimental data and theoretical expressions and, which is more important, offers critical analysis of them.

The present paper is a brief overview of the procedure of elaborating the recommended values, the socalled adjustment of the values of the fundamental physical constants. Consideration is given to the structure of data and their accuracy, the simplified procedure of adjustment, the role of relatively less appropriate data, and the reliability of the recommended values and the peculiarities of their application in the physics of the atomic nucleus and elementary particles.

\section{WHAT IS THE ADJUSTMENT OF THE FUNDAMENTAL CONSTANTS?}

It is well to begin with a clarification of what the adjustment of the fundamental constants values is. 
Recommended values of some fundamental physical constants [1]. Type of data (exact, auxiliary, independent, etc.) and the blocks they belong to related to the fine structure constant $\alpha$ or Plank constant $h$, are noted

\begin{tabular}{|c|c|c|c|}
\hline Constant & Value & $u_{r}$ & Notes \\
\hline$c$ & $299792458 \mathrm{~m} / \mathrm{s}$ & 0 & Exact* \\
\hline$\mu_{0}$ & $4 \pi \times 10^{-7} \mathrm{~N} / \mathrm{m}^{2}$ & 0 & Exact* \\
\hline$\overline{R_{\infty}}$ & $10973731.568525(73) \mathrm{m}^{-1}$ & {$\left[6.6 \times 10^{-12}\right]$} & Auxiliary ${ }^{\star}$ \\
\hline$m_{p} / m_{e}$ & $1836.15267261(85)$ & {$\left[4.6 \times 10^{-10}\right]$} & Auxiliary ${ }^{\star}$ \\
\hline$m_{e}$ & $5.4857990945(24) \times 10^{-4} \mathrm{u}$ & {$\left[4.4 \times 10^{-10}\right]$} & Auxiliary ${ }^{\star}$ \\
\hline$\overline{\alpha^{-1}}$ & $137.03599911(46)$ & {$\left[3.3 \times 10^{-9}\right]$} & $\alpha$ block $^{\star}$ \\
\hline$\chi_{C}=\hbar /\left(m_{e} c\right)$ & $386.1592678(26) \times 10^{-15} \mathrm{~m}$ & {$\left[6.7 \times 10^{-9}\right]$} & $\alpha$ block \\
\hline$h N_{\mathrm{A}}$ & $3.990312716(27) \times 10^{-10} \mathrm{~J} \mathrm{~s} / \mathrm{mol}$ & {$\left[6.7 \times 10^{-9}\right]$} & $\alpha$ block \\
\hline$R_{K}=h / e^{2}$ & $25812.807449(86) \Omega$ & {$\left[3.3 \times 10^{-9}\right]$} & $\alpha$ block $^{\star}$ \\
\hline $\bar{e}$ & $1.60217653(14) \times 10^{-19} \mathrm{C}$ & {$\left[8.5 \times 10^{-8}\right]$} & $h$ block $^{*}$ \\
\hline$h$ & $6.6260693(11) \times 10^{-34} \mathrm{~J} \mathrm{~s}$ & {$\left[1.7 \times 10^{-7}\right]$} & $h$ block $^{\star}$ \\
\hline$N_{\mathrm{A}}$ & $6.0221415(10) \times 10^{23} \mathrm{~mol}^{-1}$ & {$\left[1.7 \times 10^{-7}\right]$} & $h$ block $^{\star}$ \\
\hline$F=e N_{\mathrm{A}}$ & 96485.3383(83) C/mol & {$\left[8.6 \times 10^{-8}\right]$} & $h$ block $^{\star}$ \\
\hline$m_{e}$ & $0.510998918(44) \mathrm{MeV} / c^{2}$ & {$\left[8.6 \times 10^{-8}\right]$} & $h$ block $^{\dagger}$ \\
\hline$m_{e}$ & $9.1093826(16) \times 10^{-31} \mathrm{~kg}$ & {$\left[1.7 \times 10^{-7}\right]$} & $h$ block $^{\dagger}$ \\
\hline$K_{J}=2 e / h$ & $483597.879(41) \times 10^{9} \mathrm{~Hz} / \mathrm{V}$ & {$\left[8.5 \times 10^{-8}\right]$} & $h$ block $^{\star}$ \\
\hline$G$ & $6.6742(10) \times 10^{-11} \mathrm{~m}^{3} /\left(\mathrm{kg} \mathrm{s}^{2}\right)$ & {$\left[1.5 \times 10^{-4}\right]$} & Independent \\
\hline
\end{tabular}

Note: Designations: $u_{r}$ is a relative uncertainty; $*$ is a value in SI units recorded by definition; ${ }^{\star}$ is a measured and adjusted quantity; ${ }^{\dagger}$ is not a measured (with enough accuracy) but a derivative quantity; ${ }^{*}$ - the charge $e$ is not measured immediately, but measured in different combinations with $h$ and $N_{A}$.

There is a relatively small number of truly independent fundamental constants, such as the speed of light $c$, the magnetic vacuum constant $\mu_{0}$, the Planck constant $h$, the electron charge $e$, the Avogadro constant $N_{A}$, the masses of the electron $m_{e}$ and of the proton $m_{p}$, etc.

In a sense we can "measure" not the dimensional constants themselves but only their numerical values. The numerical values of the dimensional constants suggest units of measurement, and so we may fix some numerical values (for example, $c$ and $\mu_{0}$ values) by definition by choosing appropriate units, whereupon the other values are the subject of the measurements. As we have noted, the independent constants are relatively few in number, but experimentation allows one to measure a great variety of their combinations. So, we may measure the fine structure constant $\alpha$, Rydberg constant $R_{\infty}$, Josephson constant $K_{J}=2 e / h$, von Klitzing constant $R_{\mathrm{K}}=h / e^{2}$, Faraday constant $F=e N_{A}$, various magnetons, gyromagnetic ratios, masses of particles in terms of frequency and in atomic mass units, etc.

Thus, there occurs a great body of data on various combinations of the fundamental constants. Since nothing can be either absolutely accurate or absolutely correct, the results are not in ideal agreement and need to be "adjusted," i.e., it is necessary to produce a set of values of the fundamental constants that are of high accuracy and in conformity with each other. A substantial metrological element of this data array (hidden from the physical readers) is involvement of standards of several SI units (recall that we are dealing here in particular with numerical values of certain dimensional constants in SI units). Therefore, it is essential to adjust not only the data obtained but also the standards used for measurements.

\section{OVERVIEW OF THE BASIC DATA UNDER ADJUSTMENT}

Measurements of various quantities vary in accuracy. In fact, adjustment is needed where a number of interrelated quantities of much the same accuracy are measured. Otherwise, less accurate measurements may be rejected.

All data may be divided into five basic groups. There are two big blocks of data to be adjusted. One of them is related to the fine structure constant and the other to the Planck constant. The remaining three types of data play different parts in adjusting these two blocks. Examples of constants of different types are given in the table.

The most accurate data feasible today refer to such quantities as the Rydberg constant, masses of particles or atoms in terms of frequency or in atomic mass units, and various mass ratios. These data form a block of auxiliary data that are rather easy to handle and are pro- 
cessed largely prior to the basic adjustment. Characteristic accuracies comprise units of the 10th or 11th digit.

The data pertaining to the fine structure constant have a much lower accuracy. Therefore, high-accuracy knowledge of such constants as the Rydberg constant,

$$
R_{\infty}=\frac{\alpha^{2} m_{e} c}{2 h},
$$

plays a leading part in forming of this data block. For the Rydberg constant, the result is the constraint for the $\alpha$ and $h / m_{e}$ values and the comparison of the measurements in frequency units and in atomic mass units relates the values of the fine structure constant and the Planck molar constant $h N_{\mathrm{A}}$.

The processing of all data of this block provides us with highly accurate values

$$
\alpha=\frac{e^{2}}{4 \pi \epsilon_{0} \hbar c}
$$

and $h N_{\mathrm{A}}$, which fulfill the role of constraints for the data of the other block, corresponding to the Planck constant. The indicated constraints occur among the three fundamental constants $h, e$, and $N_{\mathrm{A}}$.

The other data play a very limited part in adjustment. Measurements of some quantities are of low accuracy and in fact neglected during adjustment, and the corresponding constants are deduced from the results of the adjustment (as, for example, the value of electron mass in kilograms). The other constants are independent of all the blocks, and their determination is therefore a mere finding of an average (as with the gravitational constant). For details, see [3, 4].

Strictly speaking, the structure of the blocks looks more intricate. The issue is that the constants enter into various equations both in an additive and in a multiplicative manner. If in the leading approximation the constants are connected by a simple relationship similar to Eqs. (1) and (2), then what matters is only the relative accuracy of the constants involved in the relationship. Thus, in Eq. (2) the quantities $c$ and $\epsilon_{0}$ are known exactly, while the measurement of $\alpha$ is more accurate than for $e$ and $h$. Using the result for the more accurate quantity $\alpha$, we can get the relationship for adjusting $e$ and $h$.

A different situation occurs when small additive corrections have a constraint of a similar type. For example, the frequencies of transitions in the hydrogen atom are defined in the main approximation by the quantity $c R_{\infty}$, but there is a wide variety of small corrections dependent on the magnitude of $\alpha$, masses of the electron and of the proton, the proton charge radius, etc. Since these corrections are small, the absolute error of their contribution to the transition frequencies is important. If the corrections are known to better absolute accuracy than the dominating contribution, then their errors may be neglected. As a result, a number of constants that hold significance only for similar small con- tributions, such as constants of weak interactions, appear among auxiliary data despite the relatively low accuracy of their measurement.

\section{THE ROLE OF "LOW-ACCURACY" MEASUREMENTS}

Ideally, not quite accurate measurements and calculations are of minor importance. As in any computation following the method of least squares, the importance of the data decreases drastically with increase in their uncertainties. However, in a nonideal case, where data adjustment is problematic, things change, and with a large body of data similar imperfect conditions emerge inevitably. In "straightforward" handling of data of the same kind, the problem of discrepancy is solved statistically. However, if the data disagree and the results of two distinct measurement methods, even though realized with different accuracy, are inconsistent, the problem cannot be resolved from the general statistical considerations. The data should be analyzed.

The main job in adjusting the constants consists actually in analyzing the data and in seeking to realize whether credence can be given to particular results, how the results correlate and what needs to be done if there are discrepancies.

Technically, the departure of a less accurate result by several standard deviations leads to the fact that, despite the low significance of the result as compared to more accurate ones, the displacement will all the same be noticeable. However, a more essential problem is that any disagreement in data casts some doubt upon whether simple statistical processing can yield reliable results.

Below we will outline the basic data blocks to see how much the corresponding data are in accordance.

\section{THE FINE STRUCTURE CONSTANT AND RELATED QUANTITIES}

The fine structure constant can be defined in a variety of ways: quantum-electrodynamic (e.g., on the basis of investigations of the electron magnetic moment), laser-quantum-mechanical (starting from the measurement of atomic recoil caused by subsequent photon absorption and emission), electrodynamic (based on a comparison of the classical electrodynamic characteristics and the characteristics of quantum effects), etc. The results are shown in Fig. 1.

The vertical band in the figure corresponds to the recommended value [1]. The results obtained using the different methods are on the whole in agreement. A specific feature of the data is that despite a great variety of measurement techniques, one or two values predominate. 


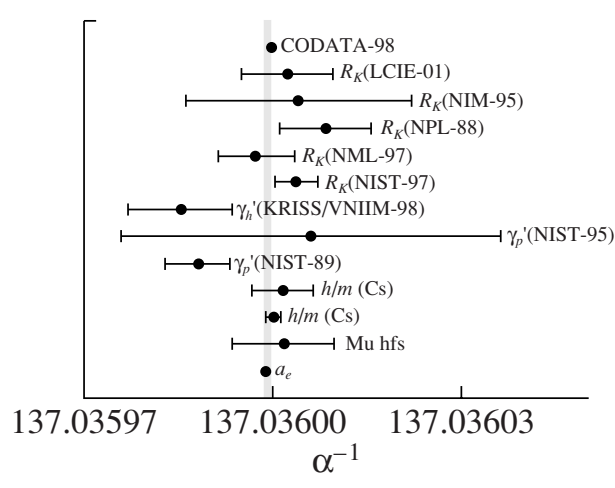

Fig. 1. Measurements of the fine structure constant. References and comprehensive explanation of different results can be found in [1].

\section{THE PLANCK CONSTANT AND RELATED CONSTANTS}

The Planck constant is determined by different methods, and their main distinction from the experiments with the fine structure constant lies in the inevitable inclusion of one or another standard. A measurement (or rather a major international project proposing numerous independent measurements of a wide variety of quantities), related to the determination of the Avogadro constant, needs a standard crystal corresponding to the so-called ideal crystal with accurately controlled corrections.

The other measurements (see Fig. 2), the best being experiments with Watt balance, are electrodynamic ones wherein the classical macroscopic effects are compared with quantum ones and the leading part belongs to the standards of voltage and resistance.

A peculiar feature of the data is that the electric results do not agree with the result based on the measurement of the Avogadro constant. The vertical band corresponds to the recommended value [1]. Its uncertainty is larger than that of more accurate original values and is due to the discrepancy in data.

\section{ELECTROMAGNETIC MEASUREMENTS AND STANDARDS OF THE BASIC ELECTRIC UNITS}

As we have mentioned above, to calculate the values of a number of fundamental constants one has often to use the standards of electric units. The current status of these standards does not reflect the general structure of the SI system. The major specific property of this field is the profound distinction in the accuracy of measurements in electrical engineering (when operating with electric circuits) and electrodynamics (when there is a need to work out electric or magnetic fields).

Electrotechnical measurements are relatively simple and allow high accuracy. However, the basis for representation of the electric units in the SI system is the

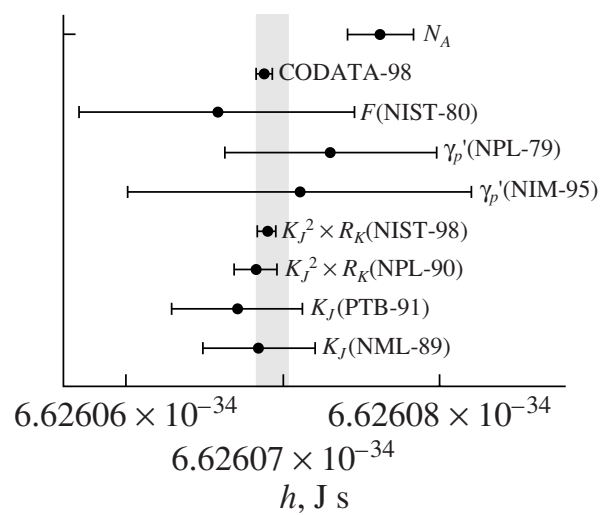

Fig. 2. Measurements of the Planck constant. References and comprehensive explanation of different results can be found in [1].

Ampere law, a law of electrodynamics. The practical realization of this law is much more cumbersome and less accurate than electrotechnical measurements. Therefore, the measurements are performed in two steps. First, there are practical units of resistance and voltage, which are used to conduct all precision measurements. Second, in certain measurements these practical units are defined in SI units, i.e., in terms of volts and ohms. Strictly speaking, just determining the values of the constants $\alpha$ and $h$ (more likely, their related quantities $R_{K}$ and $K_{J}$ ) marks such experiments (for more detail, see [4]).

Why do the Klitzing constant $R_{K}$ and the Josephson constant $K_{J}$ occur in equations? The most attractive standards are those realizable on classical macroscopic objects, yet the characteristics of which are quantized, which proves to be feasible by means of macroscopic quantum effects. The most important of those in metrology are the quantum Hall effect (resistance is quantized in terms of $R_{K}$ ) and the Josephson quantum effect (such that voltage is quantized in terms of $K_{I}$ and of some frequency under measurement).

Modern practical units of resistance and voltage are defined so that in their terms the magnitudes of $R_{K}$ and $K_{J}$ are known exactly [5]. In practice, this leads to the fact that, to convert the result of measurement for one or another quantity from the practical units to the SI system ones, this quantity should be multiplied by an appropriate combination of $R_{K}$ and $K_{J}$.

These two factors cardinally change the dependence of the measured quantity on the fundamental constants, and in some cases they are responsible for the whole of this dependence. As a rule, the experiment proper is not related to these factors and the dominant bulk of its subject matter is to construct an arrangement that can be either computed or calibrated by the methods of classical physics. The emergence of $R_{K}$ and $K_{J}$ often leads to confusion and lack of understanding of what properly is measured in the experiment. 
Thus, experiments with Watt balance measure, as is clear from the name, a macroscopic body's weight equilibrated by a force of electrodynamic origin. Highprecision determination of this force seems impracticable, and yet extra measurements in the same system but in the dynamic mode (measured is the induced emf) make it possible to arrive at the required geometric factor. Measurements of only a classical type are taken, but the quantum standards are used as the voltage and resistance standards. As a result, the classical balance leads to the quantum constant $h$.

The so-called measurements of the von Klitzing constant $R_{K}$ are organized in an analogous manner. The basic idea of the experiment is to construct a calculable capacitor, a device the capacitance and hence impedance of which for current of known frequency are known to high accuracy. Comparing the impedance with the quantum standard resistance, we can give the resistance unit in this standard (corresponding to $R_{K}$ ) with SI farad.

\section{ARE THE MEASURED FUNDAMENTAL CONSTANTS FUNDAMENTAL?}

The most fundamental constants in physics are undeniably $c, h$, and $G$. However, we have already mentioned that the constants and their numerical values are not one and the same. There is some arbitrariness in choosing numerical values (i.e., in choosing units), whereas constants are an objective phenomenon. Constants may be, for example, connected with each other in a somewhat different manner than we imagine; they may change over time; instead of one constant, there may appear a few close to each other in value (e.g., the charges of the proton and positron may in principle differ); etc.

Nevertheless, the most accurate investigations of the fundamental effects are often not related immediately to the numerical values of the fundamental constants. Constraints on the exotic effects or a practical checking of precision computations for various theories are as a rule made with a much better accuracy than that to which the corresponding physical constants are known.

The velocity of light is not an exception. We know its numerical value exactly, rather than the velocity of light itself, because the experimental realizations of a second and, to a greater extent, of a meter are limited in accuracy. Numerous experiments on checking the general and special theories of relativity are in no way related to the accuracy of length and time unit realization.

Experiments with the gravitation constant $G$ stand by themselves and have no effect on the other data to be adjusted. In spite of the fundamental nature of $G$, its measurements are not of particular importance for computation in both fundamental and practical physics. The motion of bodies in the field of the Earth's gravitational force is determined by acceleration in free fall, which is measured with high accuracy. The motion of celestial bodies requires knowledge of not the quantity $G$ itself but its product by the solar mass (and if necessary by the mass of the planets). All these products are known to much better accuracy than $G$ is. The experiments checking the general theory of relativity also deal with such products. The equivalence principle at distances in the laboratory scale may be checked in experiments similar to the $G$ measurement; however, for the equivalence principle, relative measurements are essential, which is quite a different problem. In other words, measuring $G$ is an interesting but completely isolated problem.

The Planck constant $h$ is quite another matter. The key distinction is in the existence of macroscopic quantum and discrete effects. Among the former are the quantum Hall and Josephson effects, while the latter make themselves evident in the fact that the charge and mass carriers are particles that can be counted in one way or another. Measuring the Avogadro and Faraday constants is an example of such counting. Even without recalculating the number of particles and without studying the macroscopic quantum effects, all the same we cannot avoid a known macroscopic character of manifestations of quantum mechanics. The matter is that a number of quantities like voltage are simultaneously both intensive and extensive in their character. Extensiveness manifests itself in the fact that voltage may be compared with mechanical work involving a great number of particles. As for intensity, it makes itself evident in characteristic one-particle phenomena, e.g., threshold ones in which the macroscopic potential difference matches to intra-atomic and molecular bonds.

\section{MICROSCOPIC AND MACROSCOPIC QUANTITIES}

Comparing the phenomena of the macro- and microcosm plays an important part in determining values of the fundamental physical constants. Most of the fundamental constants are quantum in nature, whereas we need practical units and want them to be useful when operating with cumbersome macroscopic objects. Meanwhile, the fundamental parameters of particles and atoms make it possible to find natural and stable parameters and, therefore, the association of the microscopic and macroscopic quantities is a key problem in metrology. Wherever the problem is solved successfully, for example in frequency and length measurements, it is possible to achieve the best measurement accuracy; but where this is impossible, macro- and microunits are used in parallel, causing often confusion.

The character of some units is evident. Thus, kilograms and amperes are macroscopic units, while atomic mass units are microscopic. Some units are more intricate in character. The scale of all the SI units, except volts, is defined by macroscopic effects, 
whereas the magnitude of a volt is immediately related to the characteristic potential difference for atomic or molecular bond breakage. However, from the metrological point of view, when we are dealing with precision measurements, of more importance is not the scale of the quantity, but the effects used in its definition. Thus, the hertz, second, and meter are microscopic units defined by atomic effects. In order for these units to be convenient in common use, large numerical coefficients are introduced, but these do not change the character of the units.

In the present-day SI version, the kilogram is a macroscopic unit because it fits the mass of a macroscopic artifact - a weight, the kilogram's prototype. The values of all the electric units in an SI are defined on the basis of the Ampere law in terms of kilograms, meters, and seconds and hence are also of macroscopic character. This applies in particular to the volt and to its derivative, the electron-volt.

It is with electron-volts that much confusion arises. The point is that, by measuring masses of atoms and particles and energies of atomic and nuclear transitions in microscopic units, one can attain an extremely high accuracy. The hertz, reciprocal meter, and atomic mass unit are microscopic units. Measurement of the same quantities in macroscopic units, such as the kilogram, joule, and electron-volt, has often a much lower precision.

It is important to be aware of the fact that if SI units are concerned, the electron-volt has no specific advantages over the joule and kilogram: the velocity of light is known with absolute accuracy, and the accuracies for measuring $h$ and $e$ agree up to a factor of 2. Conversion of more accurate values measured in hertz or other microscopic units to joules and electron-volts just requires $h$ and $e$. The fact that in precision investigations all measurements are inevitably taken either in frequency units or in relative units (like the atomic mass unit) is also fundamental. Therefore, the results for the electron or proton mass, for X-ray transition frequencies, etc. directly appear just in microscopic units and only then are converted to conventional electron-volts with loss in accuracy.

It should be noted that an immediate effort to measure energy in electron-volts will not only lead to results of lower accuracy; these attempts will culminate all the same in a result in microscopic units followed by conversion to macroscopic ones, since all precision electric measurements are taken in practical units and to convert them it is necessary to know the values of $h$ and $e$. These practical units are in essence microscopic units.

Concluding the discussion of microscopic and macroscopic units, we emphasize that a proper representation of precision data proposes that these data are given in either microscopic or macroscopic units, but uncertainties (of measurement and conversion to macro- scopic units) should necessarily be separated and a conversion factor clearly specified.

\section{THE RELIABILITY \\ OF THE RECOMMENDED VALUES}

Papers $[1,2]$ describe fully the data related to two blocks to be adjusted, allowing one to have a general picture, to appreciate how much the final recommended values depend on certain data and to determine the degree of reliability of the results from the competing independent measurements being available. Strictly speaking, handling of these blocks is adjustment in a narrow sense of this word.

Unfortunately, the situation with the so-called auxiliary data, which are known better than needed for adjustment, is more tangled. In many cases such results start from one measurement or computation, or from a series of correlated measurements and computations.

The papers [1,2] offer a comprehensive analysis of data for the Rydberg constant $R_{\infty}$ and gravitation constant $G$, whereas the description of data on proton charge radius $R_{p}$ and its $g$ factor is insufficient. The latter is not accidental, because there are no reliable independent results of sufficient accuracy pertaining to these quantities. There is for instance a body of handled data of scattering by the proton charge; however, despite the high quality of the handling itself (see, for example, [6]), there are a number of questions regarding the data (see, for example, [7]).

If the correct values of the auxiliary data are suddenly found to differ by ten and in some cases even by a hundred standard deviations, this will have no effect on the adjusted blocks. The results for $\alpha, h$, and related constants will be unaffected, and so such changes are inessential for adjustment in a narrow sense.

It should be realized that data analysis may essentially improve the quality of their processing within certain limits and the handling results may be "better" than the initial data (for example, more reliable and at times more accurate). All these possibilities are, however, limited by the initial data-both by the results of measurements and computations and by thorough analysis of them. By and large, the recommended values arising from the collectively processed results of precision experimental and theoretical studies are the most accurate and reliable interpretation of the available data.

However, with real problems for one or another constant, the recommended values may be not entirely adequate. To put it differently, whatever the data are, such are the recommended values. By way of recommendation to users, we emphasize that to assess the reliability of certain recommended values, one should find out which data these are based on. All this information is given in [1]. 


\section{HOW TO USE THE RECOMMENDED DATA}

As has repeatedly been emphasized above, in a number of cases there is a need to refer immediately to the data analysis in [1] rather than to the recommended values presented there. In the general case, proceed as follows. If a computation or measurement is not too accurate and its accuracy is incomparable to that of the recommended values [1], it is advisable to use the recommended values. The convenience lies in that all analogous calculations of various groups will use identical values of constants.

However, if the application accuracy compares favorably with the accuracy in [1], one should consult the initial data thoroughly analyzed in [1]. The best way will be not to use one or another value of a constant but to calculate it from a new measurement and compare it with the whole set of values in [1]. For instance, if a measurement is performed of a quantity with a theoretical expression sensitive to the fine structure constant's value, then the most informative way will be to compute $\alpha$ from the mentioned experiment and to compare it with the available values (see Fig. 1) rather than to compare the experiment and theory (for a certain $\alpha$ ).

\section{CONCLUSIONS}

In concluding this paper, I make two remarks. First and foremost, it should be emphasized that the main usefulness of the adjustment of fundamental constants values regularly performed by CODATA is not in the table of recommended values, which are available from many compilations, but in a comprehensive critical analysis of the initial data, which is offered only in $[1,2]$.
The adjusted data come from measurements and computations fitting a diversity of fields in physics. Each of them has its own fundamental laws and its own "standard" approximations, effective approaches and measurement methods, and standards. Adjustment of constants is a unique checking of our adequate understanding of a physical picture as a whole. Despite some moderate disagreements in the data, we may speak about their close agreement on the whole, which is to say that we appreciate adequately the world around us.

\section{ACKNOWLEDGMENTS}

The author is grateful to P.J. Mohr and V.A. Shelyuto for helpful discussions. The work was partially supported by the Russian Foundation for Basic Research (project 06-02-16156) and by DFG (project GZ 436 RUS 113/769/0).

\section{REFERENCES}

1. P. J. Mohr and B. N. Taylor, Rev. Mod. Phys. 77, 1 (2005).

2. P. J. Mohr and B. N. Taylor, Rev. Mod. Phys. 72, 351 (2000).

3. R. J. Mohr and B. N. Taylor, Phys. Today 53, 6 (Aug. 2000); Phys. Today 54, 29 (March 2001).

4. S. G. Karshenboim, Usp. Fiz. Nauk 175, 271 (2005) [Phys.-Usp. 48, 255 (2005)].

5. T. J. Quinn, Metrologia 26, 69 (1989); Metrologia 38, 89 (2001).

6. I. Sick, Phys. Lett. B 576, 62 (2003).

7. S. G. Karshenboim, Can. J. Phys. 77, 241 (1999); Phys. Rep. 422, 1 (2005). 